\title{
Analysis of Fuzzy Enhancement of Digital Image
}

\author{
Shengyi Wu \\ Jiangxi Police College, Nanchang, Jiangxi, 330103, China
}

\begin{abstract}
At present, due to the rapid development of computer technology, the overall development trend of image processing is based on digital processing (unless otherwise noted, the image processing described below defaults to digital image processing), because this method has high processing precision, Gray-scale and more, can carry out complex non-linear operations, good repeatability and many other advantages. Digital image processing is popularly refers to the application of computer and digital hardware equipment for image processing, in essence, computer technology, information theory and signal processing combined with a comprehensive application of disciplines.
\end{abstract}

Keywords: Fuzzy Enhancement, Digital Image, Technology Support

\section{Introduction}

In the natural sciences, people have long been accustomed to the pursuit of accuracy, always want to mathematically describe things, however, in the face of ambiguity, the traditional mathematical methods have encountered substantial difficulties. But for the human brain, it has a very high fuzzy division, fuzzy judgment and fuzzy reasoning ability, and people in order to express and transfer knowledge in the natural language has been cleverly infiltrated the ambiguity, and can use the least Vocabulary expresses as much information as possible. However, for the computer, no matter how it develops, can not reach the realm of the human brain, so the computer to deal with fuzzy information, you need a fuzzy language can be formalized tools, the mathematical way to deal with this fuzzy. 
L.A. Zade proposed the concept of fuzzy sets will be the general set of membership functions to the concept of extension to the fuzzy set. For the development and maturation of fuzzy mathematics has laid a solid foundation. The emergence of fuzzy set theory has aroused great interest in mathematical circles and scientific and engineering circles, and has been extensively researched, and the results of theory and application are emerging constantly, thus creating a new science) fuzzy mathematics. Fuzzy set theory is a more reasonable abstraction and description of a kind of objective things and properties, which is an inevitable extension of traditional set theory. An important feature of fuzzy mathematics is that mathematics in turn absorbs the fuzzy recognition and decision features of the human brain and applies it to the computer so that some natural language can be directly entered into the program as an algorithmic language so that people can use simple procedures to mobilize The machine accomplishes complex tasks, greatly improving machine flexibility.

\section{Analysis and Implementation of Traditional Fuzzy Theory Image Enhancement Algorithm}

In general, the image is inevitably losing information when it is mapped into a one-dimensional image, so the image is inherently fuzzy. While the human vision for the image from black to white gray level is fuzzy and difficult to accurately distinguish. This leads to ambiguities in the definition of image edges, regions, textures, and the interpretation of low-level processing results. Therefore, due to the complexity of the image itself, uncertainty and imprecision may occur at different stages of the process, namely ambiguity. In recent years, many scholars have devoted to the introduction of fuzzy set theory to image processing and recognition technology, and achieved remarkable results. Their research results show that the image processing and recognition technology based on fuzzy set theory has better effect than traditional methods in some occasions.

Shannon pointed out that the essence of information is to eliminate or reduce uncertainty. In the past, however, uncertainty was often seen as purely random, as Shannon's theory of information has made great achievements. Randomness is one of the most important uncertainties, but not the only one. In stochastic uncertainty, the set of studies generally has explicit epitaxy, that is, whether an object conforms to a concept must be either one or the other. Uncertainty is only expressed in whether or not the events represented by these objects occur, Where the main work is randomness, can use probability theory, stochastic processes and mathematical statistics tools for research. However, in many cases, there is often an extension ambiguity between the sets studied, ie, the intermediary transition property between the sets, which leads to the uncertainty of object partitioning. This uncertainty is a kind of uncertainty which is different from the randomness, called fuzzy uncertainty. In order to be able to deal with this phenomenon of uncertainty and things, many scholars have been studied. L.A. Zadeh put forward the concept of fuzzy subset, created the fuzzy mathematics, 
thus providing a set of strict mathematical methods, used to describe the phenomenon with fuzzy uncertainty and things.

\section{Analysis and Implementation of Image Enhancement Algorithm based on Fuzzy Theory}

Computer user interface refers to the computer and other users of the dialogue interface between the computer system is an important component. The development of the computer is not only the computer itself processing speed and storage capacity of the rapid increase, but also the computer user interface continuous improvement history. The importance of the user interface is that it greatly affects the use of end-users, affecting the promotion of computers and algorithms. Graphical user interface (GUI) is one of the most important achievements of computer technology today. It is convenient for non-professional users to use. It can accomplish tasks through interface operation. It is composed of window, cursor, Menu, text description and other objects constitute a user interface. The user can select, activate and manipulate these graphic objects in a certain way to perform calculations, manipulations or drawings.

MATLAB in addition to scientific computing capabilities, but also to develop the required graphical interface, Mathworks exit MATLAB6 and above version is to strengthen the graphical interface programming. Creating a MATLAB User The graphical user interface must have the following three basic elements:

Every item in MATLABGUI (buttons, labels, edit boxes, etc.) is a graphical component. Components can be divided into three categories: graphical controls (buttons, edit boxes, lists, scroll bars, etc.), static elements (windows and text strings), menus, and coordinate systems. Graphical controls and static elements are represented by the function uiContr. L Created by the functions uimenu and ui. Ontextmenu. Coordinate systems are often used to display graphical data, and are created by the axes function.

Each component of the GUI must be arranged in the image window. When you paint a data image, the image window is usually created automatically. But you can also use the function figure to create an empty image window.

If the user clicks or with the keyboard to enter some information; then the program should have the appropriate action. The mouse click or input information is an event, and if the MATLAB program runs the corresponding function, the MATLAB function will definitely react.

The realization of a GUI process includes two tasks: First, the formation of the layout of the GUI, the second is GUI component programming. In GUI, GUIDE is a component layout tool set that generates the user's required component resources and stores them in a FIG file. GUIDE generates an $\mathrm{M}$ file containing GUI initialization and release control code, which provides a callback function frame. MATLAB graphical interface program is the core of the handle graphics applications, a full understanding of the handle graphics will make MATLAB graphical interface programming easier. The handle graph is the name of a set of 
underlying graphics functions that are used to generate graphics in MATLAB and provide advanced control over graphics. The basic idea is that each visual part of MATLAB is an object, and each object has A corresponding unique identifier, the handle.

In the process of digital image processing, often faced with some complex image processing algorithms and abstract concepts, because these problems described too abstract, and the use of MATLABGUI design can solve this problem, provide an image of expression, and Can be used for the relevant algorithm using MATLAB powerful computing capabilities for further analysis and comparison, but also can perform some actions or transform to meet the needs of users or learners.

In the MATLAB main interface command window type the command guide or select the MATALB software menu bar command File $>$ New $>$ GUI pop-up graphical user interface design panel. Design panel, the left side of the control and axis, GUI Wizard provides ten kinds of control objects and an axis object. They are buttons, toggle buttons, radio buttons, edit boxes, pop-up menus, frames, static text boxes, list boxes, check boxes, and scrollbars. The axis object is used to display images, curves, etc. of the user interface. The right side of the interface provides the user area for the GUI, the user can create their own interface. Select a control, drag it to the user area with the mouse, you can draw the control. The properties of each control can be edited using the Object Attribute Editor in the upper right toolbar. For more than one control can be used in the toolbar position adjuster up and down, left and right position adjustment, to achieve the desired graphical user interface design. In the graphical user interface design panel, to create a control callback program, you can right-click on the control in the pop-up menu, select View -> CallbackS submenu, choose a way to activate the callback process, you can make the preparation process.

In accordance with the design principles of MATLABGUI, create a good GUI interface and arrange the menu, GUIDE will automatically generate $\mathrm{M}$ file to store the program's callback function, but this time only the various controls, the menu callback function prototype and comments, and no functional Function body, the user needs to be added to specific functions, adding the corresponding algorithm to complete the enhancement and other functions. The improved fast blur image enhancement algorithm itself, the choice of different $\mathrm{T}$ values, edge detection map of the effect is different. It was found that when the $\mathrm{T}$ value was less than 0.5 , when the selection of 0.2 and 0 ., the edge of the hair detail was gradually clear, but the black area appeared on the bandage of the hat: when the T value was greater than 0.5 , , The details of the edge of the hair is poor, and most of the hair area information has been blurred, but the hat on the hat and hat edge profile is clearly visible. It can be seen that when the closed-form selection is greater than 0.5 , the enhancement effect on the image information and contour information is obvious. When the threshold is less than 0.5 , the enhancement effect of the image detail information is obvious. 


\section{Conclusion}

Digital image processing technology has made great progress in recent decades, and has been widely used in scientific research and life practice. It has become a study object of computer science, information science, biology and medicine and other disciplines. Image enhancement technology, as a low-level image processing method, is usually ambiguous. The effect of image enhancement will directly affect the subsequent image analysis and pattern recognition of digital image processing. To improve the traditional fuzzy enhancement method, we can select the better image faster, but need to compare the enhanced image, humancomputer interaction to determine the parameters of change, how to automatically choose the best parameters to be further studied. There is no uniform evaluation standard for the quality of the enhanced image. For many years, people have been hoping to find a way to measure image quality as a basis for evaluating images and designing an image system to eliminate the inaccuracy of many existing qualitative test methods However, due to the current human visual system mechanism has not fully understood, although made some progress, but the problem has not been well resolved.

\section{Acknowledgement}

2014 Jiangxi Police College key disciplines funded projects

2015 Project of Scientific and Technological Research of Jiangxi Department of Education (Project No.: GJJ151185)

\section{References}

[1] Zhang Jiashu, Li Minghua. A New Image Fuzzy Enhancement Technique [J]. Journal of Southwest China Normal University (Natural Science Edition). 1994 (01)

[2] Zhang Xinrong, Wang Qitian, Yao Wenqing. Fuzzy enhancement of fingerprint image [J] .Journal of Tianjin University, 1990 (04)

[3] Wang Linyuan. Image Enhancement Based on Improved Fuzzy Image Processing [J]. Wireless Internet Technology, 2014 (12)

[4] Chen Xuguang.Fuzzy enhancement in airport recognition [J]. Fujian Computer. 2008 (09)

[5] Wang Yaming, Zheng Kai, Kang Taijie. Improved image fuzzy enhancement technique [J]. Shanghai Biomedical Engineering. 1997 (04)

[6] Chen Hua, Zhang Jing, Zhang Xiaogang, Hu Hongping. Pulution method of pulverized coal area based on illumination compensation and fuzzy enhancement [J]. Acta Automatica Sinica, 2012 